University of Nebraska - Lincoln

DigitalCommons@University of Nebraska - Lincoln

$5-15-2003$

\title{
Magnetotransport properties of $\mathrm{CrO}_{2}$ films down to single-grain sizes
}

Lu Yuan

Physics Department, University of Nebraska, lyuan2@bigred.unl.edu

Y. Ovchenkov

University of Nebraska - Lincoln

Andrei Sokolov

University of Nebraska-Lincoln, sokolov@unl.edu

C.-S. Yang

University of Nebraska - Lincoln

Bernard Doudin

University of Nebraska-Lincoln, bernard.doudin@ipcms.unistra.fr

See next page for additional authors

Follow this and additional works at: https://digitalcommons.unl.edu/physicsliou

Part of the Physics Commons

Yuan, Lu; Ovchenkov, Y.; Sokolov, Andrei; Yang, C.-S.; Doudin, Bernard; and Liou, Sy_Hwang, "Magnetotransport properties of $\mathrm{CrO}_{2}$ films down to single-grain sizes" (2003). Si-Hwang Liou Publications. 78.

https://digitalcommons.unl.edu/physicsliou/78

This Article is brought to you for free and open access by the Research Papers in Physics and Astronomy at DigitalCommons@University of Nebraska - Lincoln. It has been accepted for inclusion in Si-Hwang Liou Publications by an authorized administrator of DigitalCommons@University of Nebraska - Lincoln. 
Authors

Lu Yuan, Y. Ovchenkov, Andrei Sokolov, C.-S. Yang, Bernard Doudin, and Sy_Hwang Liou 


\title{
Magnetotransport properties of $\mathrm{CrO}_{2}$ films down to single-grain sizes
}

\author{
L. Yuan, Y. Ovchenkov, A. Sokolov, C.-S. Yang, B. Doudin, and S. H. Liou ${ }^{\text {a) }}$ \\ Center for Materials Research and Analysis and the Department of Physics and Astronomy, \\ University of Nebraska, Lincoln, Nebraska 68588-0111
}

(Presented on 12 November 2002)

\begin{abstract}
The magnetotransport properties of $\mathrm{CrO}_{2}$ films down to single-grain sizes have been investigated. The magnetoresistance of highly grain oriented $\mathrm{CrO}_{2}$ (100) films, deposited onto $\mathrm{TiO}_{2}$ (100) substrates using a high pressure cell, has been measured in the temperature range of $1.5-300 \mathrm{~K}$ and in magnetic fields up to $6 \mathrm{~T}$. The difference in the magnetoresistance at the low temperature of these samples is related to the value of the residual resistance ratio ( $\left.\mathrm{RRR}=R_{273 \mathrm{~K}} / R_{4.2 \mathrm{~K}}\right)$ of the samples. The negative magnetoresistance observed in some of these films is likely due to defects (disorder) in the $\mathrm{CrO}_{2}$ crystal. We observed a large positive longitudinal magnetoresistance in the $\mathrm{CrO}_{2}$ sample when it was measured with the current perpendicular to the $c$ axis. The nature of the large value of the longitudinal magnetoresistance may be related to the complex configuration of the Fermi surface of $\mathrm{CrO}_{2}$. (C) 2003 American Institute of Physics. [DOI: 10.1063/1.1555987]
\end{abstract}

\section{INTRODUCTION}

$\mathrm{CrO}_{2}$ is a half metallic alloy which has a high degree of spin polarization. There are many interesting spin-dependent transport properties that are due to the effect of grain boundaries, such as memory effect, low-field magenetoresistance (MR), etc. ${ }^{1-10}$ Low-field MR has been reported in polycrystalline films, ${ }^{1-6}$ epitaxial films, ${ }^{2,5,7,8}$ and powder compacts of $\mathrm{CrO}_{2} \cdot{ }^{9} \mathrm{Up}$ to $50 \% \mathrm{MR}$ has been observed on $\mathrm{CrO}_{2} / \mathrm{Cr}_{2} \mathrm{O}_{3}$ composites at low temperature. ${ }^{9}$ Measurements on these samples have shown that a disruption in the crystalline order at the grain boundaries influences the electrical conduction and results in a large low-field MR component, especially at low temperatures. However, there are still some questions that need to be addressed. For example, the opposite sign of the transverse MR was observed by different groups. Gupta's group $^{2}$ observed a positive $25 \%$ transverse MR with current parallel to $c$ axis. Watts et l. $^{3}$ observed a similar result. While the negative transverse MR was observed by Suzuki et $a$. $^{4}$ Positive MR observed at low temperatures in the epitaxial films has been attributed to the Lorentz force effect. Since the Lorentz force effect is sensitive to the mean-free electron path, the difference in reported data may be explained, for example, by different concentrations of impurities or different defects of the studied films.

In this article, we present a method for processing $\mathrm{CrO}_{2}$ films using focused ion bean (FIB) milling, and discuss MR behavior of $\mathrm{CrO}_{2}$ films with different values of the residual resistance ratio $\left(\mathrm{RRR}=R_{273 \mathrm{~K}} / R_{4.2 \mathrm{~K}}\right)$. We have studied the longitudinal and transverse MR curves of multigrain and single-grain samples measured for current parallel and perpendicular to the $c$ axis.

\section{SAMPLE PREPARATION}

The $\mathrm{CrO}_{2}$ films were deposited on single crystal rutile $\mathrm{TiO}_{2}$ (100) substrates by vapor phase transport under 100

a) Author to whom correspondence should be addressed; electronic mail: sliou@unl.edu atm of oxygen using $\mathrm{CrO}_{3}$ as the precursor. The $\mathrm{CrO}_{2}$ phase is formed at $390{ }^{\circ} \mathrm{C}$. The films exhibited needlelike grains aligned in the film plane. The x-ray diffraction data shows only the sharp (200) and (400) $\mathrm{CrO}_{2}$ peaks, in addition to the $\mathrm{TiO}_{2}$ peaks from the substrate, with no detectable $\mathrm{Cr}_{2} \mathrm{O}_{3}$ diffraction peaks. ${ }^{11}$ The existence of a thin $(\sim 2 \mathrm{~nm}) \mathrm{Cr}_{2} \mathrm{O}_{3}$ layer has been observed on our films by using x-ray photoemission spectroscopy. ${ }^{10-12}$ The magnetic properties of the films are characterized from superconducting quantum interference device measurements at room temperature and with the field applied in the sample plane. The Curie temperature of the film is about $395 \mathrm{~K}$ and the magnetic moment of the film at $300 \mathrm{~K}$ is near $2 \mu_{B}$. The coercive field $\left(H_{c}\right)$ of the film is $1.27 \mathrm{kA} / \mathrm{m}$. The thickness of the film is about $1 \mu \mathrm{m}$. The estimated size of the crystallites in the film is about 5 $\times 2 \mu \mathrm{m}^{2}{ }^{10,11}$

It has been known that to process $\mathrm{CrO}_{2}$ is difficult. Various methods have been developed to overcome this difficulty to obtain the patterns for transport measurement. ${ }^{2,3,7}$ For example, selective-area growth of $\mathrm{CrO}_{2}$ was used by Gupta. ${ }^{2}$ Patterned $\mathrm{SiO}_{2}$ was deposited on the $\mathrm{TiO}_{2}$ substrate; the $\mathrm{CrO}_{2}$ grows selectively within the stripe window openings on $\mathrm{TiO}_{2}$, but not on the adjoining $\mathrm{SiO}_{2}$ surface. In this study, we show a method for processing $\mathrm{CrO}_{2}$ films using FIB milling.

For transport measurements, we used a four-probe dc method. Current and potential leads were made by sputtering silver dots and gluing wires with silver epoxy. We used a shadow mask that allowed us to obtain two orthogonal sets of dots in an area near $2 \times 2 \mathrm{~mm}^{2}$. After wiring, we scribed $\mathrm{CrO}_{2}$ films to leave just two orthogonal lines connecting sputtered contact dots. This geometry allowed us to measure transport properties along and perpendicular to the $c$ axis simultaneously. For the single-grain sized sample, we used the FIB to mill the $\mathrm{CrO}_{2}$ thin film to get the four-probe pattern (Fig. 1), with a bridge $10 \mu \mathrm{m}$ in length and $3 \mu \mathrm{m}$ in width, with crystalline orientation along the $b$ or $c$ axis. 


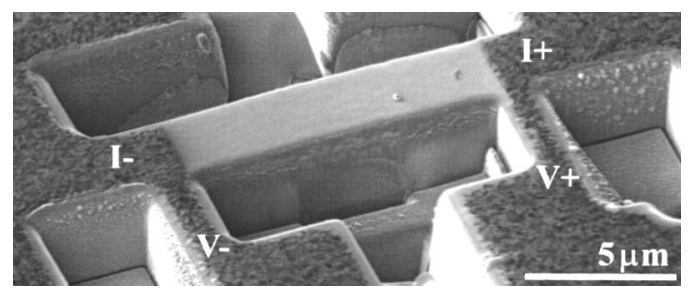

FIG. 1. Four-probe measurement pattern with $10 \mu \mathrm{m}$ in length and $3 \mu \mathrm{m}$ in width on a single grain of $\mathrm{CrO}_{2}$, milled by FIB from a film made of aligned needlelike grains. The current is flowing along the $c$ axis.

\section{RESULTS AND DISCUSSION}

Figure 2 shows the transverse magnetoresistance [MR is defined as $\left(R_{H^{-}} R_{0}\right) / R_{0}$ ] data of an FIB milled sample measured with current parallel to the $c$ axis of the crystal at different temperatures. The MR and the residual resistance values are nearly constant within temperature ranging from $1.6 \mathrm{~K}$ to $20 \mathrm{~K}$. The positive MR with a $B^{2}$ field dependence is attributed to the Lorentz force effect. With the increasing resistance of the sample at temperatures higher than $20 \mathrm{~K}$, the positive MR diminishes as it should for the Lorentz MR. At high temperatures $(\geqslant 150 \mathrm{~K})$, MR is negative and approximately linear with applied field. The origin of negative MR at high temperatures was discussed in many papers in great detail ${ }^{2,3,13}$ and was attributed to the spin-disorder scattering suppressed by the applied magnetic field. The explanation of the negative MR by magnon scattering damping is currently generally accepted.

We use the residual resistance ratio, RRR $=R_{273 \mathrm{~K}} / R_{4.2 \mathrm{~K}}$, to relate different MR behaviors of needlelike grain aligned $\mathrm{CrO}_{2}$ films. The values of RRR for all the films used in this study were calculated for the current parallel to the $c$ axis geometry. Figure 3 shows transverse MR curves at $1.6 \mathrm{~K}$ for the samples with RRR values of 11, 27, and 40, respectively. With decreasing RRR value, we observe a decrease of the MR, becoming negative for the lowest RRR values.

Since the RRR is a good characteristic for the mean-free electron path, at low temperatures, one would expect that Lorentz-type MR should become negligible for the samples

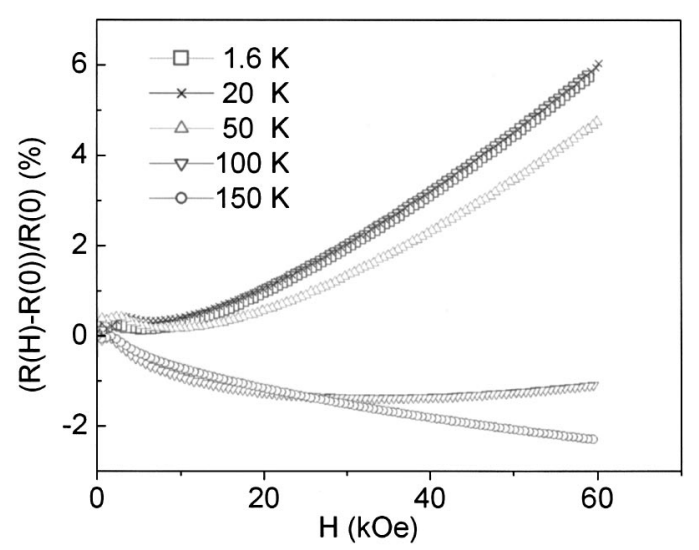

FIG. 2. High-field transverse MR, defined as $\left(R_{H^{-}} R_{0}\right) / R_{0}$, at five different temperatures for single-grain size $\mathrm{CrO}_{2}$ sample with current parallel to the $c$ axis geometry.



FIG. 3. Transverse MR vs applied magnetic field of grains aligned $\mathrm{CrO}_{2}$ films with different residual resistance ratios $\left(\mathrm{RRR}=R_{273 \mathrm{~K}} / R_{4.2 \mathrm{~K}}\right)$ measured with current parallel to $c$ axis geometry. Inset shows the magnified low-field part of MR curves for the samples.

with a low RRR value. However, the appearance of negative MR needs to take into account another source of MR. Usually the intergrain tunneling resistance is used in explaining the negative MR of granular films at low temperature. Magnetic measurements indicate that the magnetization of the samples is saturated at field of approximately $1.7 \mathrm{kOe}$. However, the shape of the MR curves corresponding to RRR $=11$ and 27, show that the negative MR becomes positive in fields $(\sim 20 \mathrm{kOe})$ much higher than the saturation field. A curve with an intermediate value of $R R R=27$ has a low-field behavior similar to the one of high RRR value (inset Fig. 3) superposed with a negative MR becoming dominant at high fields. This clearly indicates a source of MR other than intergrain tunneling.

An analysis of reported results, ${ }^{2-7}$ as well as our data, shows that at low temperature negative MR usually appears at RRR lower than 20-30 and the magnitude of negative MR increases as RRR decreases. It is logical to conclude that the origin of negative MR is the suppression of scattering on intrinsic defects (disorder) in the crystals. Further investigation of the properties of $\mathrm{CrO}_{2}$ films with special artificial defects, as introduced by deformation or radiation damage, might promote an understanding of how defect scattering is reflected by negative MR behavior in this metastable compound.

An interesting result we have observed on all measured samples is a large longitudinal MR for the case when current is perpendicular to the $c$ axis. Figure 4(a) shows the longitudinal and transverse MR curves for the sample with RRR $=40$ measured for current parallel to the $c$ axis and current perpendicular to $c$ axis. In the last case, the longitudinal MR is even higher than transverse.

To exclude the possible effects connected with nonuniform current flow due to grain boundaries and intergrain defects, we have investigated the magnetotransport properties of samples with dimensions comparable to grain sizes. Single-grain sized samples were prepared by FIB milling. We milled the $\mathrm{CrO}_{2}$ thin films to the four-probe measurement pattern (as shown in Fig. 1), with $10 \mu \mathrm{m}$ between the two potential pads and a width of about $3 \mu \mathrm{m}$, which is 

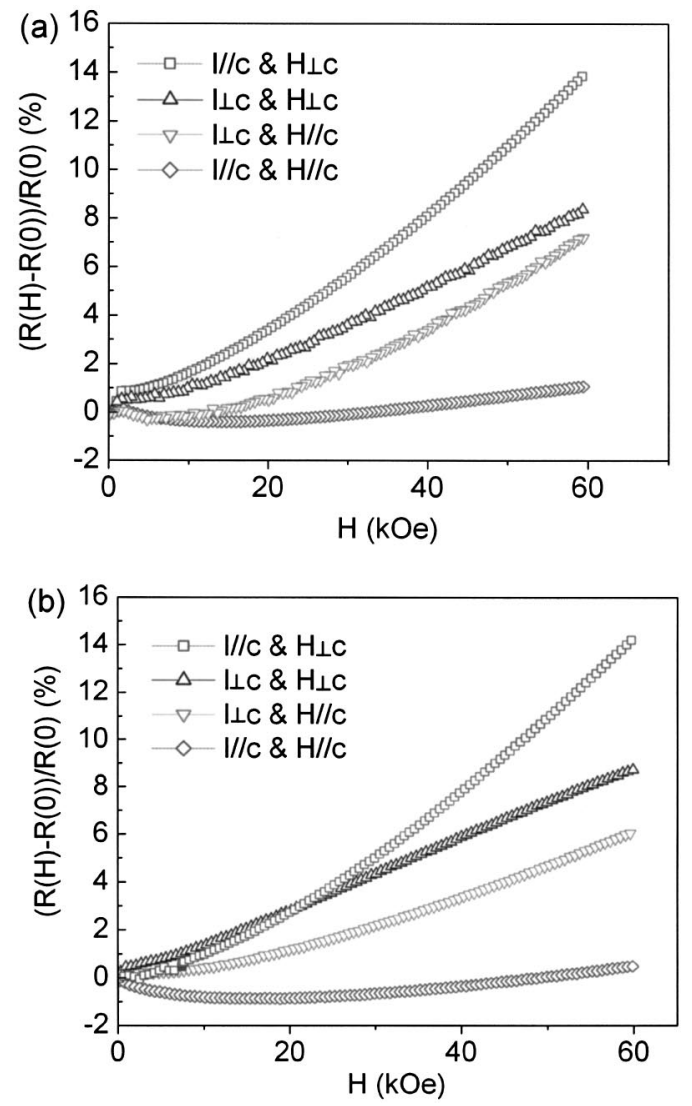

FIG. 4. Longitudinal and transverse MR for the current perpendicular and parallel to the $c$ axis direction at low temperature $(1.6 \mathrm{~K}$ ) of (a) multigrain sample with the RRR value about 40 and (b) FIB milled single-grain sample.

comparable to the single crystal size. Figure 4(b) shows both transverse and longitudinal MR curves of the sample with current parallel to $c$ axis and perpendicular to $c$ axis under applied fields up to $6 \mathrm{~T}$. When the current is perpendicular to the $c$ axis, longitudinal MR is higher than transverse MR and the shape of curve differs from the usual Lorentz-type MR curve. It is rather linear with a tendency to saturate in high fields. The results obtained on these FIB milled samples are similar to those obtained on larger samples including behavior in low fields at low temperature. Thus, the effect of nonuniform of current can be discarded.

The transverse MR proportional to $B^{2}$ and near zero longitudinal MR is the result expected for free electrons and for metals with isotropic Fermi surfaces. Therefore, to explain the large longitudinal MR in $\mathrm{CrO}_{2}$, one should take into account the geometry of the Fermi surface of this material. The calculated Fermi surface shape reported by Mazin ${ }^{14}$ consists of an electron "pseudocube" that touches hole "hammerheads" extending along $\mathbf{k}_{z}$. Calculation showed the occurrence of a finite static interband conductivity. If we consider classical electron trajectories in a magnetic field, we can see that $\mathbf{x y}$ cross sections of the Fermi surface, corre- sponding to the trajectories in the field applied along the $c$ axis, contain only pure electronic or pure hole closed orbitals, with the exception of cross sections around $z=0.4 \pi / c$, where electron and hole pockets come into contact. Cross sections of the Fermi surface with planes parallel to the $z$ axis in $\mathbf{k}$ space, corresponding to the electron trajectories in the field perpendicular to the $c$ axis, can contain both electron- and hole-type orbitals. Therefore, the large longitudinal MR for the current perpendicular to the $c$ axis may be a consequence of interband current in $\mathrm{CrO}_{2}$. Detailed theoretical calculations need to be performed for a complete description of the magnetotransport properties of $\mathrm{CrO}_{2}$.

\section{CONCLUSIONS}

Systematic studies on the MR properties of $\mathrm{CrO}_{2}$ films and single-grain samples have been presented. We show a convenient method for processing $\mathrm{CrO}_{2}$ film using FIB milling, which is convenient for patterning this metastable material and avoids wet etching process. The study of grainoriented samples with different RRR attributes the source of the low-temperature negative MR to defects (disorder) in the $\mathrm{CrO}_{2}$ crystal. These defects (disorder) may occur during the film growth. The magnitude of the low-temperature positive MR due to the Lorentz force effect is correlated to RRR as well. We report a large positive longitudinal $\mathrm{MR}$ in the $\mathrm{CrO}_{2}$ sample when the current is perpendicular to the $c$ axis, which can be related to the interband conductivity in $\mathrm{CrO}_{2}$.

\section{ACKNOWLEDGMENTS}

This work was supported by NSF CAREER program Grant No. DMR98-74657, NSF Grant No. DMR01-16780, MRSEC Grant No. DMR-0213808, the Office of Naval Research, and the Nebraska Research Initiative.

${ }^{1}$ H. Y. Hwang and S.-W. Cheong, Science 278, 1607 (1997).

${ }^{2}$ A. Gupta, X. W. Li, and G. Xiao, J. Appl. Phys. 87, 6073 (2000).

${ }^{3}$ S. M. Watts, S. Wirth, S. von Molnár, A. Barry, and J. M. D. Coey, Phys. Rev. B 61, 9621 (2000).

${ }^{4}$ K. Suzuki and P. M. Tedrow, Phys. Rev. B 58, 11597 (1998).

${ }^{5}$ K. Suzuki and P. M. Tedrow, Appl. Phys. Lett. 74, 428 (1999).

${ }^{6}$ S. S. Manoharan, D. Elefant, G. Reiss, and J. B. Goodenough, Appl. Phys. Lett. 72, 984 (1998).

${ }^{7}$ S. J. Liu, J. Y. Juang, K. H. Wu, T. M. Uen, Y. S. Gou, and J.-Y. Lin, Appl. Phys. Lett. 80, 4202 (2002).

${ }^{8}$ P. A. Stampe, R. J. Kennedy, S. M. Watts, and S. von Molnár, J. Appl. Phys. 89, 7696 (2001).

${ }^{9}$ J. M. D. Coey, A. E. Berkowitz, L. Balcells, F. F. Putris, and A. Barry, Phys. Rev. Lett. 80, 3815 (1998).

${ }^{10}$ A. Sokolov, C.-S. Yang, L. Yuan, S. H. Liou, Ruihua Cheng, B. Xu, C. N. Borca, P. A. Dowben, and B. Doudin, J. Appl. Phys. 91, 8801 (2002).

${ }^{11}$ R. Cheng, Z. Y. Liu, X. Bo, S. Adenwalla, L. Yuan, S. H. Liou, and P. A. Dowben, Mater. Lett. 56, 295 (2002).

${ }^{12}$ R. Cheng, B. Xu, C. N. Borca, A. Sokolov, C.-S. Yang, L. Yuan, S. H. Liou, B. Doudin, and P. A. Dowben, Appl. Phys. Lett. 79, 3122 (2001).

${ }^{13}$ I. I. Mazin, D. J. Singh, and C. Ambrosch-Draxl, J. Appl. Phys. 85, 6220 (1999).

${ }^{14}$ I. I. Mazin, D. J. Singh, and C. Ambrosch-Draxl, Phys. Rev. B 59, 411 (1999) 